\title{
Nonself-Adjoint Second-Order Difference Operators in Limit-Circle Cases
}

\author{
Bilender P. Allahverdiev \\ Department of Mathematics, Suleyman Demirel University, 32260 Isparta, Turkey \\ Correspondence should be addressed to Bilender P. Allahverdiev, bilender@fef.sdu.edu.tr
}

Received 4 January 2012; Accepted 11 April 2012

Academic Editor: Yuming Shi

Copyright (C) 2012 Bilender P. Allahverdiev. This is an open access article distributed under the Creative Commons Attribution License, which permits unrestricted use, distribution, and reproduction in any medium, provided the original work is properly cited.

We consider the maximal dissipative second-order difference (or discrete Sturm-Liouville) operators acting in the Hilbert space $\ell_{w}^{2}(\mathbb{Z})(\mathbb{Z}:=\{0, \pm 1, \pm 2, \ldots\})$, that is, the extensions of a minimal symmetric operator with defect index $(2,2)$ (in the Weyl-Hamburger limit-circle cases at $\pm \infty$ ). We investigate two classes of maximal dissipative operators with separated boundary conditions, called "dissipative at $-\infty$ " and "dissipative at $\infty$." In each case, we construct a self-adjoint dilation of the maximal dissipative operator and its incoming and outgoing spectral representations, which make it possible to determine the scattering matrix of the dilation. We also establish a functional model of the maximal dissipative operator and determine its characteristic function through the Titchmarsh-Weyl function of the self-adjoint operator. We prove the completeness of the system of eigenvectors and associated vectors of the maximal dissipative operators.

\section{Introduction}

Method of contour integration of the resolvent is one of the general methods of the spectral analysis of nonself-adjoint (dissipative) operators. It is related to a fine estimate of the resolvent on expanding contours which separates the spectrum. The feasibility of this method is restricted to weak perturbations of self-adjoint operators and operators having sparse discrete spectrum. Since there are no asymptotics of the solutions for a wide class of singular problems, this method cannot be applied properly.

It is well known [1-4] that the theory of dilations with application of functional models gives an adequate approach to the spectral theory of dissipative (contractive) operators. In this theory, a key role is played by the characteristic function, which carries the full information on the spectral properties of the dissipative operator. Thus, the dissipative operator becomes the model in the incoming spectral representation of the dilation. The completeness problem of the system of eigenvectors and associated vectors is solved by the factorization of the characteristic function. The computation of the characteristic functions 
of dissipative operators is preceded by the construction and investigation of the self-adjoint dilation and the corresponding scattering problem, in which the characteristic function is realized as the scattering matrix [5]. The adequacy of this approach for dissipative Jacobi operators and second-order difference (or discrete Sturm-Liouville) operators has been indicated in [6-9].

In this paper, we consider the maximal dissipative second-order difference (or discrete Sturm-Liouville) operators acting in the Hilbert space $\ell_{w}^{2}(\mathbb{Z})$, that is the extensions of a minimal symmetric operator with defect index $(2,2)$ (in the Weyl-Hamburger limit-circle cases at $\pm \infty)$. We investigate two classes of maximal dissipative operators with separated boundary conditions, called "dissipative at $-\infty$ " and "dissipative at $\infty$." In each of these cases we construct a self-adjoint dilation of the maximal dissipative operator and its incoming and outgoing spectral representations, which make it possible to determine the scattering matrix of the dilation according to the scheme of Lax and Phillips [5]. By means of the incoming spectral representation, we establish a functional model of the maximal dissipative operator and construct its characteristic function using the Titchmarsh-Weyl function of the self-adjoint operator. Finally, on the basis of the results obtained for the characteristic functions, we prove the theorems on completeness of the system of eigenvectors and associated vectors (or root vectors) of the maximal dissipative second-order difference operators.

\section{Preliminaries}

Let $y=\left\{y_{n}\right\}$ be a sequence of complex numbers $y_{n}(n \in \mathbb{Z})$ and $\ell_{1} y$ denote the sequence with components $\left(\ell_{1} y\right)_{n}$. We consider the following second-order difference (or discrete SturmLiouville) equation on the whole line:

$$
\left(\ell_{1} y\right)_{n}:=-a_{n-1} y_{n-1}+b_{n} y_{n}-a_{n} y_{n+1}=\lambda w_{n} y_{n}
$$

where $\lambda$ is a complex spectral parameter, $w_{n}>0, a_{n} \neq 0$, and $a_{n}, b_{n} \in \mathbb{R}:=(-\infty, \infty), n \in \mathbb{Z}$.

If we let $p_{n}=a_{n}, q_{n}=b_{n}-a_{n}-a_{n-1}$, and $\Delta x_{n}=x_{n+1}-x_{n}$, (2.1) can be written in Sturm-Liouville form as follows:

$$
-\Delta\left(p_{n-1} \Delta y_{n-1}\right)+q_{n} y_{n}=\lambda w_{n} y_{n}, \quad n \in \mathbb{Z}
$$

For arbitrary sequences $y=\left\{y_{n}\right\}$ and $z=\left\{z_{n}\right\}, n \in \mathbb{Z}$, we denote by $[y, z]$ the sequence with components $[y, z]_{n}$ defined as:

$$
[y, z]_{n}:=a_{n}\left(y_{n} \bar{z}_{n+1}-y_{n+1} \bar{z}_{n}\right), \quad n \in \mathbb{Z}
$$

Let $m, n \in \mathbb{Z}$ with $n<m$. Then we have the Green's formula:

$$
\sum_{j=n}^{m}\left[\left(\ell_{1} y\right)_{j} \bar{z}_{j}-y_{j}\left(\ell_{1} \bar{z}\right)_{j}\right]=[y, z]_{m}-[y, z]_{n-1} .
$$

For any sequence $y=\left\{y_{n}\right\}$, let $\ell y$ denote the sequence with components $(\ell y)_{n}$ given by $(\ell y)_{n}=\left(1 / w_{n}\right)\left(\ell_{1} y\right)_{n}, n \in \mathbb{Z}$. We denote by $\ell_{w}^{2}(\mathbb{Z})\left(w:=\left\{w_{n}\right\}, n \in \mathbb{Z}\right)$ the Hilbert space of 
all complex sequences $y=\left\{y_{n}\right\}, n \in \mathbb{Z}$ such that $\sum_{n=-\infty}^{\infty} w_{n}\left|y_{n}\right|^{2}<\infty$, with the inner product $(y, z)=\sum_{n=-\infty}^{\infty} w_{n} y_{n} \bar{z}_{n}$. Next, we denote by $D$ the set of all vectors $y \in \ell_{w}^{2}(\mathbb{Z})$ such that $\ell y \in \ell_{w}^{2}(\mathbb{Z})$. We define a maximal operator $L$ on $D$ by setting $L y=\ell y$.

It follows from Green's formula (2.4) that the limits $[y, z]_{\infty}=\lim _{n \rightarrow \infty}[y, z]_{n}$ and $[y, z]_{-\infty}=\lim _{n \rightarrow-\infty}[y, z]_{n}$ exist and are finite for arbitrary vectors $y, z \in D$. Therefore, taking the limit as $n \rightarrow-\infty$ and $m \rightarrow \infty$ in (2.4), for all $y, z \in D$, we have

$$
(L y, z)-(y, L z)=[y, z]_{\infty}-[y, z]_{-\infty} .
$$

Denote by $L_{0}$ the closure of the symmetric operator $L_{0}^{\prime}$ defined by $L_{0}^{\prime} y=L y$ on the linear set $D_{0}^{\prime}$ of finite sequences (i.e., vectors having only finitely many nonzero components) $y=\left\{y_{n}\right\}(n \in \mathbb{Z})$. The minimal operator $L_{0}$ is symmetric and $L_{0}^{*}=L$. The computation of the defect index of $L_{0}$ can be reduced to the computation of the defect index for the halfline case. In fact, $\ell_{w}^{2}(\mathbb{Z})$ is the orthogonal sum of the space $\ell_{w}^{2}\left(\mathbb{N}_{-}\right),\left(\mathbb{N}_{-}=\{-1,-2,-3, \ldots\}\right)$ and $\ell_{w}^{2}\left(\mathbb{N}_{0}\right)\left(\mathbb{N}_{0}=\{0,1,2, \ldots\}\right)$ which are imbedded in the natural way in $\ell_{w}^{2}(\mathbb{Z})$. Denote by $L_{0}^{-}\left(L_{-}\right)$and $L_{0}^{+}\left(L_{+}\right)$the minimal (maximal) operators generated by $\ell_{-}$and $\ell_{+}$in the spaces $\ell_{w}^{2}\left(\mathbb{N}_{-}\right)$and $\ell_{w}^{2}\left(\mathbb{N}_{0}\right)$, respectively, and $D_{0}^{\mp}\left(D_{\mp}\right)$ is a domain of $L_{0}^{\mp}\left(L_{\mp}\right)$, where $\left(\ell_{\mp} y\right)_{n}:=(\ell y)_{n^{\prime}}$ $n \in \mathbb{Z} \backslash\{-1,0\},\left(\ell_{-} y\right)_{-1}:=\left(1 / w_{-1}\right)\left(-a_{-2} y_{-2}+b_{-1} y_{-1}\right),\left(\ell_{+} y\right)_{0}:=\left(1 / w_{0}\right)\left(b_{0} y_{0}-a_{0} y_{1}\right)$. Then it is easy to see that the equality def $L_{0}=\operatorname{def} L_{0}^{-}+\operatorname{def} L_{0}^{+}$is satisfied for the defect number $\operatorname{def} L_{0}:=\operatorname{dim}\left\{\left(L_{0}-\lambda I\right) D\left(L_{0}\right)\right\}^{\perp}, \operatorname{Im} \lambda \neq 0$, of $L_{0}$. This shows that the defect index of $L_{0}$ has the form $(k, k)$, where $k=0,1$ or 2 . For defect index $(0,0)$ the operator $L_{0}$ is self-adjoint, that is, $L_{0}^{*}=L_{0}=L$.

Assume that the symmetric operator $L_{0}$ has defect index $(2,2)$. There are several sufficient conditions that guarantee Weyl-Hamburger limit-circle cases at $\pm \infty$ (i.e., the operator $L_{0}$ has defect index $(2,2)$, see [10-17]). The domain of $L_{0}$ consists of precisely those vectors $y \in D$ satisfying the condition

$$
[y, z]_{\infty}-[y, z]_{-\infty}=0, \quad \forall z \in D
$$

Denote by $P^{(1)}(\lambda)=\left\{P_{n}^{(1)}(\lambda)\right\}$ and $P^{(2)}(\lambda)=\left\{P_{n}^{(2)}(\lambda)\right\}, n \in \mathbb{Z}$ the solutions of $(2.1)$ satisfying the initial conditions:

$$
\begin{gathered}
P_{-1}^{(1)}(\lambda)=0, \quad P_{0}^{(1)}(\lambda)=1, \\
P_{-1}^{(2)}(\lambda)=-\frac{1}{a_{-1}}, \quad P_{0}^{(2)}(\lambda)=0 .
\end{gathered}
$$

The Wronskian of the two solutions $y=\left\{y_{n}\right\}$ and $z=\left\{z_{n}\right\}, n \in \mathbb{N}$ of (2.1) is defined as $W_{n}(y, z):=a_{n}\left(y_{n} z_{n+1}-y_{n+1} z_{n}\right)$, so that $W_{n}(y, z)=[y, \bar{z}]_{n}, n \in \mathbb{Z}$. The Wronskian of the two solutions of (2.1) is independent of $n$, and the two solutions of this equation are linearly independent if and only if their Wronskian is nonzero. It follows from the conditions (2.7) and the constancy of the Wronskian that $W_{n}\left(P^{(1)}, P^{(2)}\right)=1, n \in \mathbb{Z}$. Consequently, $P^{(1)}(\lambda)$ and $P^{(2)}(\lambda)$ form a fundamental system of solutions of $(2.1)$, and $P^{(1)}(\lambda), P^{(2)}(\lambda) \in \ell_{w}^{2}(\mathbb{Z})$ for all $\lambda \in \mathbb{C}$. The theory of difference equations can be seen in $[18,19]$.

Let $u=P^{(1)}(0)$ and $v=P^{(2)}(0)$. Since the vectors $u=\left\{u_{n}\right\}$ and $v=\left\{v_{n}\right\} \quad(n \in \mathbb{Z})$ are real valued and $[u, v]_{n}=1(n \in \mathbb{Z})$, the following assertion can be verified easily using (2.3). 
Lemma 2.1. For arbitrary vectors $y=\left\{y_{n}\right\} \in D$ and $z=\left\{z_{n}\right\} \in D$, one has the equality:

$$
[y, z]_{n}=[y, u]_{n}[\bar{z}, v]_{n}-[y, v]_{n}[\bar{z}, u]_{n}, \quad(n \in \mathbb{Z} \cup\{-\infty, \infty\}) .
$$

The domain $D_{0}$ of the operator $L_{0}$ consists of precisely those vectors $y \in D$ satisfying the boundary conditions:

$$
[y, u]_{-\infty}=[y, v]_{-\infty}=[y, u]_{\infty}=[y, v]_{\infty}=0 .
$$

Let us consider the following linear maps from $D$ into $\mathbb{C}^{2}$

$$
\Gamma_{1} y=\left(\begin{array}{c}
{[y, v]_{-\infty}} \\
{[y, u]_{\infty}}
\end{array}\right), \quad \Gamma_{2} y=\left(\begin{array}{c}
{[y, u]_{-\infty}} \\
{[y, v]_{\infty}}
\end{array}\right), \quad y \in D .
$$

Then we have the following result (see [8]).

Theorem 2.2. For any contraction $K$ in $\mathbb{C}^{2}$ the restriction of the operator $L$ to the set of vectors $y \in D$ satisfying the boundary conditions

$$
(K-I) \Gamma_{1} y+i(K+I) \Gamma_{2} y=0,
$$

or

$$
(K-I) \Gamma_{1} y-i(K+I) \Gamma_{2} y=0
$$

is, respectively, a maximal dissipative or a accretive extension of the operator $L_{0}$. Conversely, every maximal dissipative (accretive) extension of $L_{0}$ is the restriction of $L$ to the set of vectors $y \in D$ satisfying (2.11) (2.12), and the contraction $K$ is uniquely determined by the extension. These conditions give a self-adjoint extension if and only if $K$ is unitary. In the latter case (2.11) and (2.12) are equivalent to the condition $(\cos S) \Gamma_{1} y-(\sin S) \Gamma_{2} y=0$, where $S$ is a self-adjoint (Hermitian matrix) operator in $\mathbb{C}^{2}$. The general form of dissipative and accretive extensions of the operator $L_{0}$ is given by the conditions

$$
\begin{array}{ll}
K\left(\Gamma_{1} y+i \Gamma_{2} y\right)=\Gamma_{1} y-i \Gamma_{2} y, & \Gamma_{1} y+i \Gamma_{2} y \in D(K), \\
K\left(\Gamma_{1} y-i \Gamma_{2} y\right)=\Gamma_{1} y+i \Gamma_{2} y, & \Gamma_{1} y-i \Gamma_{2} y \in D(K),
\end{array}
$$

respectively, where $K$ is a linear operator in $\mathbb{C}^{2}$ with $\|K f\| \leq\|f\|, f \in D(K) \subseteq \mathbb{C}^{2}$. The general form of symmetric extensions is given by the formulae (2.13), where $K$ is an isometric operator.

In particular, if $K$ is a diagonal matrix, the boundary conditions

$$
\begin{gathered}
{[y, v]_{-\infty}-h_{1}[y, u]_{-\infty}=0} \\
{[y, u]_{\infty}-h_{2}[y, v]_{\infty}=0}
\end{gathered}
$$


with $\operatorname{Im} h_{1} \geq 0$ or $h_{1}=\infty$, and $\operatorname{Im} h_{2} \geq 0$ or $h_{2}=\infty\left(\operatorname{Im} h_{1} \leq 0\right.$ or $h_{1}=\infty$, and $\operatorname{Im} h_{2} \leq 0$ or $\left.h_{2}=\infty\right)$ describe all the maximal dissipative (maximal accretive) extensions of $L_{0}$ with separated boundary conditions. The self-adjoint extensions of $L_{0}$ are obtained precisely when $\operatorname{Im} h_{1}=0$ or $h_{1}=\infty$, and $\operatorname{Im} h_{2}=0$ or $h_{2}=\infty$. Here for $h_{1}=\infty\left(h_{2}=\infty\right)$ condition (2.14) (2.15) should be replaced by $[y, u]_{-\infty}=0\left([y, v]_{\infty}=0\right)$.

In what follows, we will study the dissipative operators $L_{h_{1} h_{2}}^{\mp}$ generated by $\ell$ and the boundary conditions (2.14) and (2.15) of two types: "dissipative at $-\infty$," that is, when either $\operatorname{Im} h_{1}>0$ and $\operatorname{Im} h_{2}=0$ or $h_{2}=\infty$; "dissipative at $\infty$," when $\operatorname{Im} h_{1}=0$ or $h_{1}=\infty$ and $\operatorname{Im} h_{2}>0$.

\section{Self-Adjoint Dilations of the Maximal Dissipative Operators}

In order to construct a self-adjoint dilation of the maximal dissipative operator $L_{h_{1} h_{2}}^{-}$in the case of "dissipative at $-\infty$ " (i.e., $\operatorname{Im} h_{1}>0$ and $\operatorname{Im} h_{2}=0$ or $h_{2}=\infty$ ), we associate with $H:=\ell_{w}^{2}(\mathbb{Z})$ the "incoming" and "outgoing" channels $D_{-}:=L^{2}(-\infty, 0)$ and $D_{+}:=L^{2}(0, \infty)$, we form the orthogonal sum $\mathscr{\ell}=D_{-} \oplus H \oplus D_{+}$and we call it the main Hilbert space of the dilation. In the space $\mathscr{L}$, we consider the operator $\mathcal{L}_{h_{1} h_{2}}^{-}$generated by the expression

$$
\mathcal{L}\left\langle\varphi_{-}, y, \varphi_{+}\right\rangle=\left\langle i \frac{d \varphi_{-}}{d \xi}, \ell y, i \frac{d \varphi_{+}}{d \varsigma}\right\rangle
$$

on the set $D\left(\mathcal{L}_{h_{1} h_{2}}^{-}\right)$of vectors $\left\langle\varphi_{-}, y, \varphi_{+}\right\rangle$satisfying the conditions $\varphi_{-} \in W_{2}^{1}(-\infty, 0), \varphi_{+} \in$ $W_{2}^{1}(0, \infty), y \in D$ and

$$
\begin{gathered}
{[y, v]_{-\infty}-h_{1}[y, u]_{-\infty}=\alpha \varphi_{-}(0), \quad[y, v]_{-\infty}-\bar{h}_{1}[y, u]_{-\infty}=\alpha \varphi_{+}(0),} \\
{[y, u]_{\infty}-h_{2}[y, v]_{\infty}=0}
\end{gathered}
$$

where $W_{2}^{1}$ denotes the Sobolev space and $\alpha^{2}:=2 \operatorname{Im} h_{1}, \alpha>0$.

Theorem 3.1. The operator $\mathcal{L}_{h_{1} h_{2}}^{-}$is self-adjoint in $\mathscr{H}$ and it is a self-adjoint dilation of the maximal dissipative operator $L_{h_{1} h_{2}}^{-}$.

Proof. We assume that $f, g \in D\left(\mathcal{L}_{h_{1} h_{2}}\right)$ with $f=\left\langle\varphi_{-}, y, \varphi_{+}\right\rangle$and $g=\left\langle\psi_{-}, z, \psi_{+}\right\rangle$. Then using integration by parts and (3.1), we obtain

$$
\begin{aligned}
\left(\mathcal{L}_{h_{1} h_{2}}^{-} f, g\right)_{d \mathscr{l}} & =\int_{0}^{\infty} i \varphi_{-}^{\prime} \bar{\psi}_{-} d \xi+(L y, z)_{H}+\int_{0}^{\infty} i \varphi_{+}^{\prime} \bar{\psi}_{+} d \xi \\
& =i \varphi_{-}(0) \bar{\psi}_{-}(0)-i \varphi_{+}(0) \bar{\psi}_{+}(0)+[y, z]_{\infty}-[y, z]_{-\infty}+\left(f, \mathcal{L}_{h_{1} h_{2}}^{-} g\right)_{\mathscr{\ell}}
\end{aligned}
$$

If we use the boundary conditions (3.2) for the components of the vectors $f, g$ and Lemma 2.1, we see that $i \varphi_{-}(0) \bar{\psi}_{-}(0)-i \varphi_{+}(0) \bar{\psi}_{+}(0)+[y, z]_{\infty}-[y, z]_{-\infty}=0$. Thus, $\mathcal{L}_{h_{1} h_{2}}^{-}$is symmetric. 
Therefore, to prove that $\mathcal{L}_{h_{1} h_{2}}^{-}$is self-adjoint, it is sufficient to show that $\left(\mathcal{L}_{h_{1} h_{2}}^{-}\right)^{*} \subseteq \mathcal{L}_{h_{1} h_{2}}^{-}$. Let us take $g=\left\langle\psi_{-}, z, \psi_{+}\right\rangle \in D\left(\left(\mathcal{L}_{h_{1} h_{2}}^{-}\right)^{*}\right)$ and let $\left(\mathcal{L}_{h_{1} h_{2}}^{-}\right)^{*} g=g^{*}=\left\langle\psi_{-}^{*}, z^{*}, \psi_{+}^{*}\right\rangle \in \mathscr{d}$ so that

$$
\left(\mathcal{L}_{h_{1} h_{2}}^{-} f, g\right)_{\mathscr{H}}=\left(f, g^{*}\right)_{\mathscr{H}^{\prime}} \quad \forall f \in D\left(\mathcal{L}_{h_{1} h_{2}}^{-}\right) .
$$

If we choose the components of $f \in D\left(\mathcal{L}_{h_{1} h_{2}}^{-}\right)$properly in (3.4), it becomes easy to show that $\psi_{-} \in W_{2}^{1}(-\infty, 0), \psi_{+} \in W_{2}^{1}(0, \infty), z \in D$, and $g^{*}=\mathcal{L} g$, where the operator $\mathcal{L}$ is given by (3.1). As a result, (3.4) takes the form $(\mathcal{L} f, g)_{\mathscr{L}}=\left(f, \mathcal{L g}_{\mathscr{\ell}}\right.$, for all $f \in D\left(\mathcal{L}_{h_{1} h_{2}}^{-}\right)$. Hence, in the bilinear form $(\mathcal{L} f, g)_{\mathscr{L}}$, the sum of the integral terms must be equal to zero:

$$
i \varphi_{-}(0) \bar{\psi}_{-}(0)-i \varphi_{+}(0) \bar{\psi}_{+}(0)+[y, z]_{\infty}-[y, z]_{-\infty}=0,
$$

for all $f=\left\langle\varphi_{-}, y, \varphi_{+}\right\rangle \in D\left(\mathcal{L}_{h_{1} h_{2}}^{-}\right)$. In addition, if we solve the boundary conditions (3.2) for $[y, u]_{-\infty}$ and $[y, v]_{-\infty}$, we get

$$
[y, u]_{-\infty}=-\frac{i}{\alpha}\left(\varphi_{+}(0)-\varphi_{-}(0)\right), \quad[y, v]_{-\infty}=\alpha \varphi_{-}(0)-\frac{i h_{1}}{\alpha}\left(\varphi_{+}(0)-\varphi_{-}(0)\right) .
$$

It follows from Lemma 2.1 and (3.6) that (3.5) is equivalent to the following equality:

$$
\begin{aligned}
i \varphi_{-}(0) \bar{\psi}_{-}(0)-i \varphi_{+}(0) \bar{\psi}_{+}(0)= & {[y, z]_{-\infty}-[y, z]_{\infty} } \\
= & -\frac{i}{\alpha}\left(\varphi_{+}(0)-\varphi_{-}(0)\right)[\bar{z}, v]_{-\infty} \\
& -\alpha\left[\varphi_{-}(0)-\frac{i h_{1}}{\alpha^{2}}\left(\varphi_{+}(0)-\varphi_{-}(0)\right)\right][\bar{z}, u]_{-\infty} \\
& -[y, u]_{\infty}[\bar{z}, v]_{\infty}+[y, v]_{\infty}[\bar{z}, u]_{\infty} \\
= & -\frac{i}{\alpha}\left(\varphi_{+}(0)-\varphi_{-}(0)\right)[\bar{z}, v]_{-\infty} \\
& -\alpha\left[\varphi_{-}(0)-\frac{i h_{1}}{\alpha^{2}}\left(\varphi_{+}(0)-\varphi_{-}(0)\right)\right][\bar{z}, u]_{-\infty} \\
& +\left([\bar{z}, u]_{\infty}-h_{2}[\bar{z}, v]_{\infty}\right)[y, v]_{\infty} .
\end{aligned}
$$

Note that the values $\varphi_{ \pm}(0)$ can be any complex numbers. Therefore, when we compare the coefficients of $\varphi_{ \pm}(0)$ on the left and right of the last equality we see that the vector $g=\left\langle\psi_{-}, z, \psi_{+}\right\rangle$satisfies the boundary conditions $[z, v]_{-\infty}-h_{1}[z, u]_{-\infty}=\alpha \psi_{-}(0),[z, v]_{-\infty}-$ $\bar{h}_{1}[z, u]_{-\infty}=\alpha \psi_{+}(0),[z, u]_{\infty}-h_{2}[z, v]_{\infty}=0$. Consequently, we obtain $\left(\mathcal{L}_{h_{1} h_{2}}^{-}\right)^{*} \subseteq \mathcal{L}_{h_{1} h_{2}}^{-}$and hence $\mathcal{L}_{h_{1} h_{2}}^{-}=\left(\mathcal{L}_{h_{1} h_{2}}^{-}\right)^{*}$.

The self-adjoint operator $\mathcal{L}_{h_{1} h_{2}}^{-}$generates in $\mathscr{L}$ a unitary group $U_{t}^{-}=\exp \left[i \mathcal{L}_{h_{1} h_{2}}^{-} t\right]$, $t \in \mathbb{R}$. Let $P: \mathscr{d} \rightarrow H$ and $P_{1}: H \rightarrow \mathscr{H}$ denote the mappings acting according to the formulas $P:\left\langle\varphi_{-}, y, \varphi_{+}\right\rangle \rightarrow y$ and $P_{1}: y \rightarrow\langle 0, y, 0\rangle$. Let $Z_{t}^{-}=P U_{t}^{-} P_{1} t \geq 0$. The family $\left\{Z_{t}^{-}\right\}$, $t \geq 0$, of operators is a strongly continuous semigroup of completely nonunitary contractions on $H$. (We recall that the linear bounded operator $A$ acting in the Hilbert space $H$ is called 
completely nonunitary if invariant subspace $M \subseteq H(M \neq\{0\}$ of operator $A$ whose restriction to $M$ is unitary, does not exist). Let us denote by $A_{h_{1} h_{2}}$ the generator of this semigroup: $A_{h_{1} h_{2}} y=$ $\lim _{t \rightarrow+0}(i t)^{-1}\left(Z_{t}^{-} y-y\right)$. The domain of $A_{h_{1} h_{2}}$ consists of all the vectors for which the limit exists. $A_{h_{1} h_{2}}$ is a maximal dissipative operator. The operator $\mathcal{L}_{h_{1} h_{2}}^{-}$is called the self-adjoint dilation of $A_{h_{1} h_{2}}$ [1-4]. We show that $A_{h_{1} h_{2}}=L_{h_{1} h_{2}}^{-}$, and thus $\mathcal{L}_{h_{1} h_{2}}^{-}$is a self-adjoint dilation of $L_{h_{1} h_{2}}^{-}$. To do this, we first verify the equality $[1-4]$ :

$$
P\left(\mathcal{L}_{h_{1} h_{2}}^{-}-\lambda I\right)^{-1} P_{1} y=\left(L_{h_{1} h_{2}}^{-}-\lambda I\right)^{-1} y, \quad y \in H, \operatorname{Im} \lambda<0 .
$$

Denote $\left(\mathcal{L}_{h_{1} h_{2}}^{-}-\lambda I\right)^{-1} P_{1} y=g=\left\langle\psi_{-}, z, \psi_{+}\right\rangle$. Then $\left(\mathcal{L}_{h_{1} h_{2}}^{-}-\lambda I\right) g=P_{1} y$, and hence $L z-\lambda z=y$, $\psi_{-}(\xi)=\psi_{-}(0) e^{-i \lambda \xi}$ and $\psi_{+}(\varsigma)=\psi_{+}(0) e^{-i \lambda_{\zeta}}$. Since $g \in D\left(\mathcal{L}_{h_{1} h_{2}}^{-}\right)$, and hence, $\psi_{-} \in L^{2}(-\infty, 0)$; it follows that $\psi_{-}(0)=0$, and, consequently, $z$ satisfies the boundary conditions $[z, v]_{-\infty}-$ $h_{1}[z, u]_{-\infty}=0,[z, u]_{\infty}-h_{2}[z, v]_{\infty}=0$. Therefore, $z \in D\left(L_{h_{1} h_{2}}^{-}\right)$and since a point $\lambda$ with $\operatorname{Im} \lambda<0$ cannot be an eigenvalue of a dissipative operator, it follows that $z=\left(L_{h_{1} h_{2}}^{-}-\lambda I\right)^{-1} y$. Note that $\psi_{+}(0)$ is obtained from the formula $\psi_{+}(0)=\alpha^{-1}\left([z, v]_{-\infty}-\bar{h}_{1}[z, u]_{-\infty}\right)$. Then

$$
\left(\mathcal{L}_{h_{1} h_{2}}^{-}-\lambda I\right)^{-1} P_{1} y=\left\langle 0,\left(L_{h_{1} h_{2}}^{-}-\lambda I\right)^{-1} y, \alpha^{-1}\left([z, v]_{-\infty}-\bar{h}_{1}[z, u]_{-\infty}\right) e^{-i \lambda \varsigma}\right\rangle,
$$

for $y \in H$ and $\operatorname{Im} \lambda<0$. By applying $P$, one can obtain (3.8).

Now, it is not difficult to show that $A_{h}=L_{h}^{-}$. In fact, it follows from (3.8) that

$$
\begin{aligned}
\left(L_{h_{1} h_{2}}^{-}-\lambda I\right)^{-1} & =P\left(\mathcal{L}_{h_{1} h_{2}}^{-}-\lambda I\right)^{-1} P_{1}=-i P \int_{0}^{\infty} U_{t}^{-} e^{-i \lambda t} d t P_{1} \\
& =-i \int_{0}^{\infty} Z_{t}^{-} e^{-i \lambda t} d t=\left(A_{h_{1} h_{2}}-\lambda I\right)^{-1}, \quad \operatorname{Im} \lambda<0,
\end{aligned}
$$

and thus $L_{h_{1} h_{2}}^{-}=A_{h_{1} h_{2}}$. Theorem 3.1. is proved.

In order to construct a self-adjoint dilation of the maximal dissipative operator $L_{h_{1} h_{2}}^{+}$ in the case "dissipative at $\infty$ " (i.e., $\operatorname{Im} h_{1}=0$ or $h_{1}=\infty$ and $\operatorname{Im} h_{2}>0$ ) in $\mathscr{t}$, we consider the operator $\mathcal{L}_{h_{1} h_{2}}^{+}$generated by the expression (3.1) on the set $D\left(\mathcal{L}_{h_{1} h_{2}}^{+}\right)$of vectors $\left\langle\varphi_{-}, y, \varphi_{+}\right\rangle$ satisfying the conditions $\varphi_{-} \in W_{2}^{1}(-\infty, 0), \varphi_{+} \in W_{2}^{1}(0, \infty), y \in D$ and

$$
\begin{gathered}
{[y, v]_{-\infty}-h_{1}[y, u]_{-\infty}=0, \quad[y, u]_{\infty}-h_{2}[y, v]_{\infty}=\alpha \varphi_{-}(0),} \\
{[y, u]_{\infty}-\bar{h}_{2}[y, v]_{\infty}=\alpha \varphi_{+}(0)}
\end{gathered}
$$

where $\alpha^{2}:=2 \operatorname{Im} h_{2}, \alpha>0$.

The proof of the next theorem is similar to that of Theorem 3.1.

Theorem 3.2. The operator $\mathcal{L}_{h_{1} h_{2}}^{+}$is self-adjoint in $\mathcal{d e}$ and it is a self-adjoint dilation on the maximal dissipative operator $L_{h_{1} h_{2}}^{+}$. 


\section{Scattering Theory of the Dilations and Functional Models of the Maximal Dissipative Operators}

The unitary group $U_{t}^{ \pm}=\exp \left[i \mathcal{L}_{h_{1} h_{2}}^{ \pm} t\right](t \in \mathbb{R})$ has a crucial property which enables us to apply the Lax-Phillips scheme [5]. In other words, it has incoming and outgoing subspaces $D_{-}=\left\langle L^{2}(-\infty, 0), 0,0\right\rangle$ and $D_{+}=\left\langle 0,0, L^{2}(0, \infty)\right\rangle$ satisfying the following properties:

(1) $U_{t}^{ \pm} D_{-} \subset D_{-}, t \leq 0$ and $U_{t}^{ \pm} D_{+} \subset D_{+}, t \geq 0$;

(2) $\bigcap_{t \leq 0} U_{t}^{ \pm} D_{-}=\bigcap_{t \geq 0} U_{t}^{ \pm} D_{+}=\{0\}$;

(3) $\overline{\bigcup_{t \geq 0} U_{t}^{ \pm} D_{-}}=\overline{\bigcup_{t \leq 0} U_{t}^{ \pm} D_{+}}=\mathscr{H}$;

(4) $D_{-} \perp D_{+}$.

Property (4) is obvious. To verify property (1) for $D_{+}$(the proof for $D_{-}$is similar), we set $R_{\lambda}^{ \pm}=\left(\mathcal{L}_{h_{1} h_{2}}^{ \pm}-\lambda I\right)^{-1}$, for all $\lambda$ with $\operatorname{Im} \lambda<0$. Then, for any $f=\left\langle 0,0, \varphi_{+}\right\rangle \in D_{+}$, we have

$$
R_{\lambda}^{ \pm} f=\left\langle 0,0,-i e^{-i \lambda \varsigma} \int_{0}^{\varsigma} e^{-i \lambda s} \varphi_{+}(s) d s\right\rangle
$$

Hence, we find $R_{\curlywedge} f \in D_{+}$. Therefore, if $g \perp D_{+}$, then it follows that

$$
0=\left(R_{\lambda}^{ \pm} f, g\right)_{\mathscr{d}}=-i \int_{0}^{\infty} e^{-i \lambda t}\left(U_{t}^{ \pm} f, g\right)_{\mathscr{d}} d t, \quad \operatorname{Im} \lambda<0 .
$$

From this, we conclude that $\left(U_{t}^{ \pm} f, g\right)_{\mathscr{d}}=0$ for all $t \geq 0$. Hence $U_{t}^{ \pm} D_{+} \subset D_{+}$, for $t \geq 0$, which completes the proof of property (1).

To prove property (2), we denote by $P^{+}: \mathscr{H} \rightarrow L^{2}(0, \infty)$ and $P_{1}^{+}: L^{2}(0, \infty) \rightarrow D_{+}$the mappings acting according to the formulas $P^{+}:\left\langle\varphi_{-}, u, \varphi_{+}\right\rangle \rightarrow \varphi_{+}$and $P_{1}^{+}: \varphi \rightarrow\langle 0,0, \varphi\rangle$, respectively. Note that the semigroup of isometries $V_{t}^{ \pm}=P^{+} U_{t}^{ \pm} P_{1}^{+}, t \geq 0$ is a one-sided shift in $L^{2}(0, \infty)$. Indeed, the generator of the semigroup of the one-sided shift $V_{t}$ in $L^{2}(0, \infty)$ is the differential operator $i(d / d \xi)$ satisfying the boundary condition $\varphi(0)=0$. On the other hand, the generator $A^{ \pm}$of the semigroup of isometries $V_{t}^{ \pm}, t \geq 0$, is the operator $A^{ \pm} \varphi=P^{+} \mathcal{L}_{h_{1} h_{2}}^{ \pm} P_{1}^{+} f=P^{+} \mathcal{L}_{h_{1} h_{2}}^{ \pm}\langle 0,0, \varphi\rangle=P^{+}\langle 0,0, i(d \varphi / d \xi)\rangle=i(d \varphi / d \xi)$, where $\varphi \in W_{2}^{1}(0, \infty)$ and $\varphi(0)=0$. As a semigroup is uniquely determined by its generator, it follows that $V_{t}^{ \pm}=V_{t}$, and thus, $\bigcap_{t \geq 0} U_{t}^{ \pm} D_{+}=\left\langle 0,0, \bigcap_{t \geq 0} V_{t} L^{2}(0, \infty)\right\rangle=\{0\}$, which verifies the property (2).

The scattering matrix is defined in terms of the spectral representations theory in this scheme of the Lax-Phillips scattering theory. We will continue with their construction and prove property ( 3 ) of the incoming and outgoing subspaces along the way.

We recall that the linear operator $A$ (with domain $D(A)$ ) acting in the Hilbert space $H$ is called completely nonself-adjoint (or simple) if the invariant subspace $M \subseteq D(A)(M \neq\{0\})$ of the operator $A$ whose restriction to $M$ is self-adjoint, does not exist.

Lemma 4.1. The operator $L_{h_{1} h_{2}}^{ \pm}$is completely nonself-adjoint (simple).

Proof. Let $H^{\prime} \subset H$ be a nontrivial subspace where $L_{h_{1} h_{2}}^{-}$(the proof for $L_{h_{1} h_{2}}^{+}$is similar) induces a self-adjoint operator $L^{\prime}$ with domain $D\left(L^{\prime}\right)=H^{\prime} \cap D\left(L_{h_{1} h_{2}}^{-}\right)$. If $f \in D\left(L^{\prime}\right)$, then we get $f \in D\left(L^{\prime *}\right)$ and $[y, u]_{-\infty}-h_{1}[y, v]_{-\infty}=0,[y, u]_{-\infty}-\bar{h}_{1}[y, v]_{-\infty}=0$. It follows that $[y, u]_{-\infty}=0$, $[y, v]_{-\infty}=0$ and $y(\lambda)=0$ for the eigenvectors $y(\lambda)$ of the operator $L_{h_{1} h_{2}}^{-}$that lie in $H^{\prime}$ and are 
eigenvectors of $L^{\prime}$. Since all solutions of (2.1) belong to $\ell_{w}^{2}(\mathbb{Z})$, we conclude that the resolvent $R_{\lambda}\left(L_{h_{1} h_{2}}^{-}\right)$of the operator $L_{h_{1} h_{2}}^{-}$is a Hilbert-Schmidt operator, and hence the spectrum of $L_{h_{1} h_{2}}^{-}$ is purely discrete. Using the theorem on expansion in eigenvectors of the self-adjoint operator $L^{\prime}$, we see that $H^{\prime}=\{0\}$, that is, the operator $L_{h_{1} h_{2}}^{-}$is simple. The lemma is proved.

To prove property (3) we first set

$$
\mathscr{H}_{-}^{ \pm}=\overline{\bigcup_{t \geq 0} U_{t}^{ \pm} D_{-}}, \quad \mathscr{H}_{+}^{ \pm}=\overline{\bigcup_{t \leq 0} U_{t}^{ \pm} D_{+}}
$$

and prove the following lemma.

Lemma 4.2. The equality $\mathscr{\ell}_{-}^{ \pm}+\mathscr{\ell}_{+}^{ \pm}=\mathscr{H}$ holds.

Proof. Using property (1) of the subspace $D_{ \pm}$, we can easily show that the subspace $\mathscr{L}_{ \pm}^{\prime}=$ $\mathscr{H} \ominus\left(\mathscr{L}_{-}^{ \pm}+\mathscr{H}_{+}^{ \pm}\right)$is invariant with respect to the group $\left\{U_{t}^{ \pm}\right\}$and has the form $\mathscr{H}_{ \pm}^{\prime}=\left\langle 0, H_{ \pm}^{\prime}, 0\right\rangle$, where $H_{ \pm}^{\prime}$ is a subspace in $H$. Accordingly, if the subspace $\mathscr{L}_{ \pm}^{\prime}$ (and thus, $H_{ \pm}^{\prime}$ as well) were nontrivial, then the unitary group $\left\{U_{t}^{ \pm \prime}\right\}$, restricted to this subspace, would be a unitary part of the group $\left\{U_{t}^{ \pm}\right\}$, and thus the restriction $L_{h_{1} h_{2}}^{ \pm \prime}$ of $L_{h_{1} h_{2}}^{ \pm}$to $H_{ \pm}^{\prime}$ would be a self-adjoint operator in $H_{ \pm}^{\prime}$. It follows from the simplicity of the operator $L_{h_{1} h_{2}}^{ \pm}$that $H_{ \pm}^{\prime}=\{0\}$, that is, $\mathscr{L}_{ \pm}^{\prime}=\{0\}$. The proof is completed.

Let $\varphi(\lambda)$ and $\psi(\lambda)$ be the solutions of (2.1) satisfying the conditions:

$$
\begin{gathered}
{[\varphi, u]_{-\infty}=-1, \quad[\varphi, v]_{-\infty}=0,} \\
{[\psi, u]_{-\infty}=0, \quad[\psi, v]_{-\infty}=1 .}
\end{gathered}
$$

The Titchmarsh-Weyl function $m_{\infty h_{2}}(\lambda)$ of the self-adjoint operator $L_{\infty h_{2}}^{-}$is determined by the condition $\left[\psi+m_{\infty} h_{2} \varphi, u\right]_{\infty}-h_{2}\left[\psi+m_{\infty h_{2}} \varphi, v\right]_{\infty}=0$. Then, we have

$$
m_{\infty h_{2}}(\lambda)=-\frac{[\psi, u]_{\infty}-h_{2}[\psi, v]_{\infty}}{[\varphi, u]_{\infty}-h_{2}[\varphi, v]_{\infty}} .
$$

The last equality implies that $m_{\infty h_{2}}(\lambda)$ is a meromorphic function on the complex plane $\mathbb{C}$ with a countable number of poles on the real axis, which coincide with the eigenvalues of the self-adjoint operator $L_{\infty h_{2}}$. One can also show that the function $m_{\infty h_{2}}(\lambda)$ has the following properties: $\operatorname{Im} \lambda \operatorname{Im} m_{\infty h_{2}}(\lambda)>0$ for $\operatorname{Im} \lambda \neq 0$ and $m_{\infty h_{2}}(\bar{\lambda})=\overline{m_{\infty h_{2}}(\lambda)}$ for complex $\lambda$ with the exception of the real poles of $m_{\infty h_{2}}(\lambda)$.

We adopt the following notations: $\theta(\lambda)=\psi(\lambda)+m_{\infty h_{2}}(\lambda) \varphi(\lambda)$,

$$
S_{h_{1} h_{2}}^{-}(\lambda)=\frac{m_{\infty h_{2}}(\lambda)-h_{1}}{m_{\infty h_{2}}(\lambda)-\bar{h}_{1}} .
$$

Let

$$
U_{\lambda}^{-}(\xi, \varsigma)=\left\langle e^{-i \lambda \xi},\left(m_{\infty h_{2}}(\lambda)-h_{1}\right)^{-1} \alpha \theta(\lambda), \bar{S}_{h_{1} h_{2}}^{-}(\lambda) e^{-i \lambda \varsigma}\right\rangle
$$


For real values of $\lambda$, the vectors $U_{\lambda}^{-}(\xi, \varsigma)$ do not belong to the space $\mathscr{\ell}$, but they satisfy the equation $\mathcal{L U}=\lambda U$ and the boundary conditions (3.2). Using $U_{\lambda}^{-}(\xi, \varsigma)$, we define the transformation $F_{-}: f \rightarrow \tilde{f}_{-}(\lambda)$ by $\left(F_{-} f\right)(\lambda):=\tilde{f}_{-}(\lambda):=(1 / \sqrt{2 \pi})\left(f, U_{\lambda}^{-}\right)_{\mathscr{d}}$ on the vector $f=$ $\left\langle\varphi_{-}, y, \varphi_{+}\right\rangle$, where $\varphi_{-}, \varphi_{+}$are smooth, compactly supported functions, and $y=\left\{y_{n}\right\}, n \in \mathbb{Z}$, is a finite sequence.

Lemma 4.3. The transformation $F_{-}$isometrically maps $\mathscr{H}_{-}$onto $L^{2}(\mathbb{R})$. For all vectors $f, g \in \mathscr{H}_{-}^{-}$, the Parseval equality and the inversion formula hold:

$$
(f, g)_{d \ell}=\left(\tilde{f}_{-}, \tilde{g}_{-}\right)_{L^{2}}=\int_{-\infty}^{\infty} \tilde{f}_{-}(\lambda) \overline{\tilde{g}_{-}(\lambda)} d \lambda, \quad f=\frac{1}{\sqrt{2 \pi}} \int_{-\infty}^{\infty} \tilde{f}_{-}(\lambda) U_{\lambda}^{-} d \lambda,
$$

where $\tilde{f}_{-}(\lambda)=\left(F_{-} f\right)(\lambda)$ and $\tilde{g}_{-}(\lambda)=\left(F_{-} g\right)(\lambda)$.

Proof. For $f, g \in D_{-}, f=\left\langle\varphi_{-}, 0,0\right\rangle, g=\left\langle\psi_{-}, 0,0\right\rangle$, we have

$$
\tilde{f}_{-}(\lambda):=\frac{1}{\sqrt{2 \pi}}\left(f, U_{\lambda}^{-}\right)_{\mathscr{d}}=\frac{1}{\sqrt{2 \pi}} \int_{-\infty}^{0} \varphi_{-}(\xi) e^{i \lambda \xi} d \xi \in H_{-}^{2}
$$

and, by the usual Parseval equality for Fourier integrals,

$$
(f, g)_{d \ell}=\int_{-\infty}^{0} \varphi_{-}(\xi) \overline{\psi_{-}(\xi)} d \xi=\int_{-\infty}^{\infty} \tilde{f}_{-}(\lambda) \overline{\widetilde{g}_{-}(\lambda)} d \lambda=\left(F_{-} f, F_{-} g\right)_{L^{2}}
$$

From now on, let $H_{ \pm}^{2}$ denote the Hardy classes in $L^{2}(\mathbb{R})$ consisting of the functions which are analytically extendable to the upper and lower half-planes, respectively.

Let us extend the Parseval equality to the whole $\mathscr{\ell}_{-}^{-}$. To this end, we consider in $\mathscr{\ell}_{-}^{-}$ the dense set $\mathscr{H}_{-}^{\prime}$ of vectors obtained from the smooth, compactly supported functions in $D_{-}$: $f \in \mathscr{H}_{-}^{\prime}$ if $f=U_{T}^{-} f_{0}, f_{0}=\left\langle\varphi_{-}, 0,0\right\rangle, \varphi_{-} \in C_{0}^{\infty}(-\infty, 0)$, where $T=T_{f}$ is a nonnegative number (depending on $f$ ). In this case, if $f, g \in \mathscr{L}_{-}^{\prime}$, then $U_{-T}^{-} f, U_{-T}^{-} g \in D_{-}$for $T>T_{f}$ and $T>T_{g}$. Furthermore, the first components of these vectors belong to $C_{0}^{\infty}(-\infty, 0)$. Since the operators $U_{t}^{-}, t \in \mathbb{R}$, are unitary, the equality $F_{-} U_{-T}^{-} f=\left(U_{-T}^{-} f, U_{\lambda}^{-}\right)_{\mathscr{L}}=e^{-i \lambda T}\left(f, U_{\lambda}^{-}\right)_{\mathscr{d}}=e^{-i \lambda T} F_{-} f$ gives us that

$$
\begin{aligned}
(f, g)_{\mathscr{d}} & =\left(U_{-T}^{-} f, U_{-T}^{-} g\right)_{\mathscr{d}}=\left(F_{-} U_{-T}^{-} f, F_{-} U_{-T}^{-} g\right)_{L^{2}} \\
& =\left(e^{-i \lambda T} F_{-} f, e^{-i \lambda T} F_{-} g\right)_{L^{2}}=\left(F_{-} f, F_{-} g\right)_{L^{2}} .
\end{aligned}
$$

If we take the closure in (4.11), we get the Parseval equality for the whole space $\mathscr{t}_{-}^{-}$. The inversion formula follows from the Parseval equality if all integrals in it are considered as limits in the mean of integrals over finite intervals. In conclusion, we have $F_{-} \mathscr{L}_{-}^{-}=$ $\overline{\bigcup_{t \geq 0} F_{-} U_{t}^{-} D_{-}}=\overline{\bigcup_{t \geq 0} e^{-i \lambda t} H_{-}^{2}}=L^{2}(\mathbb{R})$ which implies that $F_{-}$maps $\mathscr{L}_{-}^{-}$onto the whole of $L^{2}(\mathbb{R})$. The lemma is proved. 
Now, we let

$$
U_{\lambda}^{+}(\xi, \varsigma)=\left\langle S_{h_{1} h_{2}}^{-}(\lambda) e^{-i \lambda \xi},\left(m_{\infty h_{2}}^{-}(\lambda)-\bar{h}_{1}\right)^{-1} \alpha \theta(\lambda), e^{-i \lambda \varsigma}\right\rangle .
$$

Note as in the previous case that the vectors $U_{\lambda}^{+}(\xi, \varsigma)$, for real values of $\lambda$, do not belong to the space $\mathscr{H}$. But, $U_{\lambda}^{+}(\xi, \varsigma)$ satisfies the equation $\mathcal{L U}=\lambda U, \lambda \in \mathbb{R}$, and the boundary conditions (3.2). By means of $U_{\lambda}^{+}(\xi, \varsigma)$, we consider the transformation $F_{+}: f \rightarrow \tilde{f}_{+}(\lambda)$ by setting $\left(F_{+} f\right)(\lambda):=\tilde{f}_{+}(\lambda):=(1 / \sqrt{2 \pi})\left(f, U_{\lambda}^{+}\right)_{\mathscr{\ell}}$ on vectors $f=\left\langle\varphi_{-}, y, \varphi_{+}\right\rangle$, where $\varphi_{-}, \varphi_{+}$are smooth, compactly supported functions, and $y=\left\{y_{n}\right\}, n \in \mathbb{Z}$, is a finite sequence. The proof of the next result is similar to that of Lemma 4.3.

Lemma 4.4. The transformation $F_{+}$isometrically maps $\mathfrak{H}_{+}^{-}$onto $L^{2}(\mathbb{R})$, and for all vectors $f, g \in \mathfrak{L}_{+}^{-}$, the Parseval equality and the inversion formula hold:

$$
(f, g)_{\mathscr{H}}=\left(\tilde{f}_{+}, \tilde{g}_{+}\right)_{L^{2}}=\int_{-\infty}^{\infty} \tilde{f}_{+}(\lambda) \overline{\tilde{g}_{+}(\lambda)} d \lambda, \quad f=\frac{1}{\sqrt{2 \pi}} \int_{-\infty}^{\infty} \tilde{f}_{+}(\lambda) U_{\lambda}^{+} d \lambda,
$$

where $\tilde{f}_{+}(\lambda)=\left(F_{+} f\right)(\lambda)$ and $\tilde{g}_{+}(\lambda)=\left(F_{+} g\right)(\lambda)$.

From (4.6), we see that $\left|S_{h_{1} h_{2}}^{-}(\lambda)\right|=1$ for all $\lambda \in \mathbb{R}$. Therefore, it follows from the explicit formula for the vectors $U_{\lambda}^{+}$and $U_{\lambda}^{-}$that

$$
U_{\lambda}^{-}=\bar{S}_{h_{1} h_{2}}(\lambda) U_{\lambda^{\prime}}^{+}, \quad(\lambda \in \mathbb{R}) .
$$

Lemmas 4.3 and 4.4 imply that $\mathscr{H}_{-}^{-}=\mathscr{H}_{+}^{-}$. Together with Lemma 4.2 , this results in $\mathscr{H}^{-}=\mathscr{l}_{-}^{-}=$ $\mathscr{H}_{+}^{-}$and the property (3) of the incoming and outgoing subspaces for $U_{t}^{-}$.

Therefore, the transformation $F_{-}$maps isometrically onto $L^{2}(\mathbb{R})$ with the subspace $D_{-}$ mapped onto $H_{-}^{2}$ and the operators $U_{t}^{-}$are transformed into the operators of multiplication by $e^{i \lambda t}$, that is, $F_{-}$is the incoming spectral representation for the group $\left\{U_{t}^{-}\right\}$. Similarly $F_{+}$is the outgoing spectral representation for $\left\{U_{t}^{-}\right\}$. It is seen from (4.14) that we can realize the passage from the $F_{+}$-representation of a vector $f \in \mathscr{d}$ to its $F_{-}$-representation multiplying by the function $S_{h_{1} h_{2}}^{-}(\lambda): \tilde{f}_{-}(\lambda)=S_{h_{1} h_{2}}^{-}(\lambda) \tilde{f}_{+}(\lambda)$. According to [5], the scattering function (matrix) of the group $\left\{U_{t}^{-}\right\}$with respect to the subspaces $D_{-}$and $D_{+}$, is the coefficient by which the $F_{-}$-representation of a vector $f \in \mathscr{l}$ must be multiplied in order to get the corresponding $F_{+}$-representation: $\tilde{f}_{+}(\lambda)=\bar{S}_{h_{1} h_{2}}^{-}(\lambda) \tilde{f}_{-}(\lambda)$ and, thus, we have proved the following theorem.

Theorem 4.5. The function $\bar{S}_{h_{1} h_{2}}^{-}(\lambda)$ is the scattering matrix of the group $\left\{U_{t}^{-}\right\}$(of the self-adjoint operator $\mathcal{L}_{h_{1} h_{2}}^{-}$).

Let $S(\lambda)$ be an arbitrary nonconstant inner function [1-4] on the upper half-plane (the analytic function $S(\lambda)$ on the upper half-plane $\mathbb{C}_{+}$is called inner function on $\mathbb{C}_{+}$if $|S(\lambda)| \leq 1$ for $\lambda \in \mathbb{C}_{+}$and $|S(\lambda)|=1$ for almost all $\lambda \in \mathbb{R}$ ). Let $\mathcal{K}=H_{+}^{2} \ominus S H_{+}^{2}$. We can see that $\mathcal{K} \neq\{0\}$ is a subspace of the Hilbert space $H_{+}^{2}$. Now, let us consider the semigroup of the operators $Z_{t}, t \geq 0$, acting in $\mathcal{K}$ according to the formula $Z_{t} \varphi=P\left[e^{i \lambda t} \varphi\right], \varphi:=\varphi(\lambda) \in \mathcal{K}$, where $P$ 
denotes the orthogonal projection from $H_{+}^{2}$ onto $\mathcal{K}$. The generator of the semigroup $\left\{Z_{t}\right\}$ is denoted by $T: T \varphi=\lim _{t \rightarrow+0}(i t)^{-1}\left(Z_{t} \varphi-\varphi\right)$, which is a maximal dissipative operator acting in $\mathcal{K}$ with the domain $D(T)$ consisting of all vectors $\varphi \in \mathcal{K}$, so that the limit exists. The operator $T$ is called a model dissipative operator (note that this model dissipative operator, which is associated with the names of Lax and Phillips [5], is a special case of a more general model dissipative operator constructed by Sz-Nagy and Foiaş [1,2]). The basic assertion is that $S(\lambda)$ is the characteristic function of the operator $T$.

Let $K=\langle 0, H, 0\rangle$ so that $\mathscr{d}=D_{-} \oplus K \oplus D_{+}$. It can be concluded from the explicit form of the unitary transformation $F_{-}$that

$$
\begin{gathered}
\mathscr{H} \longrightarrow L^{2}(\mathbb{R}), \quad f \longrightarrow \tilde{f}_{-}(\lambda)=\left(F_{-} f\right)(\lambda), \\
D_{-} \longrightarrow H_{-\prime}^{2} \quad D_{+} \longrightarrow S_{h_{1} h_{2}}^{-} H_{+}^{2} \\
K \longrightarrow H_{+}^{2} \ominus S_{h_{1} h_{2}}^{-} H_{+}^{2}, \quad U_{t}^{-} f \longrightarrow\left(F_{-} U_{t}^{-} F_{-}^{-1} \tilde{f}_{-}\right)(\lambda)=e^{i \lambda t} \tilde{f}_{-}(\lambda) .
\end{gathered}
$$

The formulas (4.15) show that the operator $L_{h_{1} h_{2}}^{-}$is unitarily equivalent to the model dissipative operator with the characteristic function $S_{h_{1} h_{2}}^{-}(\lambda)$. Since the characteristic functions of unitarily equivalent dissipative operators coincide [1-4], we have proved the theorem below.

Theorem 4.6. The characteristic function of the maximal dissipative operator $L_{h_{1} h_{2}}^{-}$coincides with the function $S_{h_{1} h_{2}}^{-}(\lambda)$ defined in (4.6).

If $m_{h_{1} \infty}(\lambda)$ is the Titchmarsh-Weyl function of the self-adjoint operator $L_{h_{1} \infty}$, then it can be expressed in terms of the Wronskian of the solutions as follows:

$$
m_{h_{1} \infty}(\lambda)=-\frac{[x, v]_{\infty}}{[\phi, v]_{\infty}}
$$

Here $\phi(\lambda)$ and $\chi(\lambda)$ are solutions of (2.1) and normalized by

$$
\begin{aligned}
& {[\phi, u]_{-\infty}=-\frac{1}{\sqrt{1+h_{1}^{2}}}, \quad[\phi, v]_{-\infty}=-\frac{h_{1}}{\sqrt{1+h_{1}^{2}}},} \\
& {[x, u]_{-\infty}=\frac{h_{1}}{\sqrt{1+h_{1}^{2}}}, \quad[x, v]_{-\infty}=\frac{1}{\sqrt{1+h_{1}^{2}}} .}
\end{aligned}
$$

Let us adopt the following notations:

$$
\begin{gathered}
n(\lambda):=\frac{[\phi, u]_{\infty}}{[\chi, v]_{\infty}}, \quad m(\lambda):=m_{h_{1} \infty}(\lambda) \\
S^{+}(\lambda):=S_{h_{1} h_{2}}^{+}(\lambda):=\frac{m(\lambda) n(\lambda)-h_{2}}{m(\lambda) n(\lambda)-\bar{h}_{2}}
\end{gathered}
$$


Let

$$
V_{\lambda}^{-}(\xi, \varsigma)=\left\langle e^{-i \lambda \xi}, \alpha m(\lambda)\left[\left(m(\lambda) n(\lambda)-h_{2}\right)[\chi, v]_{\infty}\right]^{-1} \phi(\lambda), \bar{S}^{+}(\lambda) e^{-i \lambda \varsigma}\right\rangle .
$$

One can see that the vector $V_{\lambda}^{-}(\xi, \varsigma)$ does not belong to $\mathscr{H}$ for $\lambda \in \mathbb{R}$, but $V_{\lambda}^{-}$satisfies the equation $\mathcal{L} V=\lambda V, \lambda \in \mathbb{R}$, and the boundary conditions (3.11). By means of $V_{\lambda}^{-}$, we define the transformation $F_{-}: f \rightarrow \tilde{f}_{-}(\lambda)$ by $\left(F_{-} f\right)(\lambda):=\tilde{f}_{-}(\lambda):=(1 / \sqrt{2 \pi})\left(f, V_{\lambda}^{-}\right)_{\mathscr{d}}$ on the vector $f=\left\langle\varphi_{-}, y, \varphi_{+}\right\rangle$, where $\varphi_{-}, \varphi_{+}$are smooth, compactly supported functions, and $y=\left\{y_{n}\right\}, n \in$ $\mathbb{Z}$, is a finite sequence. The next result can be proved following the steps similar to the proof of Lemma 4.3.

Lemma 4.7. The transformation $F_{-}$isometrically maps $\mathfrak{L}_{-}^{+}$onto $L^{2}(\mathbb{R})$. For all vectors $f, g \in \mathfrak{H}_{-}^{+}$, the Parseval equality and the inversion formula hold:

$$
(f, g)_{\mathscr{d}}=\left(\tilde{f}_{-}, \tilde{g}_{-}\right)_{L^{2}}=\int_{-\infty}^{\infty} \tilde{f}_{-}(\lambda) \overline{\tilde{g}_{-}(\lambda)} d \lambda, \quad f=\frac{1}{\sqrt{2 \pi}} \int_{-\infty}^{\infty} \tilde{f}_{-}(\lambda) U_{\lambda}^{-} d \lambda,
$$

where $\tilde{f}_{-}(\lambda)=\left(F_{-} f\right)(\lambda)$ and $\tilde{g}_{-}(\lambda)=\left(F_{-} g\right)(\lambda)$.

Let

$$
V_{\lambda}^{+}(\xi, \varsigma)=\left\langle S^{+}(\lambda) e^{-i \lambda \xi}, \alpha m(\lambda)\left[\left(m(\lambda) n(\lambda)-\bar{h}_{2}\right)[\chi, v]_{\infty}\right]^{-1} \phi(\lambda), e^{-i \lambda \varsigma}\right\rangle .
$$

The vector $V_{\lambda}^{+}(\xi, \varsigma)$ does not belong to $\mathscr{d}$ for $\lambda \in \mathbb{R}$. However, $V_{\lambda}^{+}$satisfies the equation $\mathcal{L} V=\lambda V, \lambda \in \mathbb{R}$, and the boundary conditions (3.11). Using $V_{\lambda}^{+}(\xi, \varsigma)$, let us consider the transformation $F_{+}: f \rightarrow \tilde{f}_{+}(\lambda)$ on vectors $f=\left\langle\varphi_{-}, y, \varphi_{+}\right\rangle$, in which $\varphi_{-}, \varphi_{+}$are smooth, compactly supported functions, and $y=\left\{y_{n}\right\}, n \in \mathbb{Z}$, is a finite sequence, by setting $\left(F_{+} f\right)(\lambda):=\tilde{f}_{+}(\lambda):=(1 / \sqrt{2 \pi})\left(f, U_{\lambda}^{+}\right)_{\mathscr{\ell}}$.

Lemma 4.8. The transformation $F_{+}$isometrically maps $\mathscr{\ell}_{+}^{+}$onto $L^{2}(\mathbb{R})$. For all vectors $f, g \in \mathscr{\ell}_{+}^{+}$, the Parseval equality and the inversion formula hold:

$$
(f, g)_{d \ell}=\left(\tilde{f}_{+}, \tilde{g}_{+}\right)_{L^{2}}=\int_{-\infty}^{\infty} \tilde{f}_{-}(\lambda) \overline{\tilde{g}_{-}(\lambda)} d \lambda, \quad f=\frac{1}{\sqrt{2 \pi}} \int_{-\infty}^{\infty} \tilde{f}_{+}(\lambda) U_{\lambda}^{+} d \lambda,
$$

where $\tilde{f}_{+}(\lambda)=\left(F_{+} f\right)(\lambda)$ and $\tilde{g}_{+}(\lambda)=\left(F_{+} g\right)(\lambda)$.

It is seen from (4.18) that the function $S_{h_{1} h_{2}}^{+}(\lambda)$ satisfies $\left|S_{h_{1} h_{2}}^{+}(\lambda)\right|=1$ for $\lambda \in \mathbb{R}$. Therefore, the explicit formula for the vectors $U_{\lambda}^{+}$and $U_{\lambda}^{-}$gives us that

$$
V_{\lambda}^{-}=\bar{S}_{h_{1} h_{2}}^{+}(\lambda) V_{\lambda}^{+}, \quad \lambda \in \mathbb{R}
$$


Hence, we conclude the equality $\mathscr{L}_{-}^{+}=\mathscr{L}_{+}^{+}$from Lemmas 4.7 and 4.8. Together with Lemma 4.2, we get $\mathscr{l}^{\prime}=\mathscr{l}_{-}^{+}=\mathscr{\ell}_{+}^{+}$. We can see from (4.23) that the passage from the $F_{-}-$ representation of a vector $f \in \mathscr{l}$ to its $F_{+}$-representation is realized as follows: $\tilde{f}_{+}(\lambda)=$ $\bar{S}_{h_{1} h_{2}}^{+}(\lambda) \tilde{f}_{-}(\lambda)$. Thus, we have proved the following assertion.

Theorem 4.9. The function $\bar{S}_{h_{1} h_{2}}^{+}(\lambda)$ is the scattering matrix of the group $\left\{U_{t}^{+}\right\}$(of the self-adjoint operator $\mathcal{L}_{h_{1} h_{2}}^{+}$).

Using the explicit form of the unitary transformation $F_{-}$, we obtain

$$
\begin{gathered}
\mathscr{H} \longrightarrow L^{2}(\mathbb{R}), \quad f \longrightarrow \tilde{f}_{-}(\lambda)=\left(F_{-} f\right)(\lambda), \\
D_{-} \longrightarrow H_{-\prime}^{2} \quad D_{+} \longrightarrow S_{h_{1} h_{2}}^{+} H_{+}^{2} \\
K \longrightarrow H_{+}^{2} \ominus S_{h_{1} h_{2}}^{+} H_{+}^{2} \quad U_{t}^{+} f \longrightarrow\left(F_{-} U_{t}^{+} F_{-}^{-1} \tilde{f}_{-}\right)(\lambda)=e^{i \lambda t} \tilde{f}_{-}(\lambda) .
\end{gathered}
$$

We conclude from (4.24) that the operator $L_{h_{1} h_{2}}^{+}$is a unitary equivalent to the model dissipative operator with characteristic function $S_{h_{1} h_{2}}^{+}(\lambda)$, which in turn proves the next theorem.

Theorem 4.10. The characteristic function of the maximal dissipative operator $L_{h_{1} h_{2}}^{+}$coincides with the function $S_{h_{1} h_{2}}^{+}(\lambda)$ defined by (4.18).

\section{Completeness Theorems for the System of Eigenvectors and Associated Vectors of the Maximal Dissipative Operators}

We know that the characteristic function of a maximal dissipative operator $L_{h_{1} h_{2}}^{ \pm}$carries complete information about the spectral properties of this operator [1-4]. For example, completeness of the system of eigenvectors and associated vectors of the maximal dissipative operators $L_{h_{1} h_{2}}^{ \pm}$is guaranteed by the absence of a singular factor of the characteristic function $S_{h_{1} h_{2}}^{ \pm}(\lambda)$ in the factorization $S_{h_{1} h_{2}}^{ \pm}(\lambda)=S^{ \pm}(\lambda) B^{ \pm}(\lambda)$ (where $B^{ \pm}(\lambda)$ is a Blaschke product).

Let $A$ be a linear operator in the Hilbert space $\mathbf{H}$ with the domain $D(A)$. The complex number $\lambda_{0}$ is called an eigenvalue of the operator $A$ if there exists a nonzero element $y_{0} \in$ $D(A)$ satisfying $A y_{0}=\lambda_{0} y_{0}$. Such an element $y_{0}$ is called the eigenvector of the operator $A$ corresponding to the eigenvalue $\lambda_{0}$. The elements $y_{1}, y_{2}, \ldots, y_{k}$ are called the associated vectors of the eigenvector $y_{0}$ if they belong to $D(A)$ and satisfy $A y_{j}=\lambda_{0} y_{j}+y_{j-1}, j=1,2, \ldots, k$. The element $y \in D(A), y \neq 0$ is called a root vector of the operator $A$ corresponding to the eigenvalue $\lambda_{0}$, if all powers of $A$ are defined on this element and $\left(A-\lambda_{0} I\right)^{m} y=0$ for some integer $m$. The set of all root vectors of $A$ corresponding to the same eigenvalue $\lambda_{0}$ with the vector $y=0$ forms a linear set $N_{\lambda_{0}}$ and is called the root lineal. The dimension of the lineal $N_{\lambda_{0}}$ is called the algebraic multiplicity of the eigenvalue $\lambda_{0}$. The root lineal $N_{\lambda_{0}}$ coincides with the linear span of all eigenvectors and associated vectors of $A$ corresponding to the eigenvalue $\lambda_{0}$. Therefore, the completeness of the system of all eigenvectors and associated vectors of $A$ is equivalent to the completeness of the system of all root vectors of this operator.

Theorem 5.1. For all values of $h_{1}$ with $\operatorname{Im} h_{1}>0$, except possibly for a single value $h_{1}=h_{1}^{0}$, and for fixed $h_{2}\left(\operatorname{Im} h_{2}=0\right.$ or $\left.h_{2}=0\right)$, the characteristic function $S_{h_{1} h_{2}}^{-}(\lambda)$ of the maximal dissipative 
operator $L_{h_{1} h_{2}}^{-}$is a Blaschke product and the spectrum of $L_{h_{1} h_{2}}^{-}$is purely discrete and belongs to the open upper half plane. The operator $L_{h_{1} h_{2}}^{-}\left(h_{1} \neq h_{1}^{0}\right)$ has a countable number of isolated eigenvalues with finite algebraic multiplicity and limit points at infinity, and the system of all eigenvectors and associated vectors (or root vectors) of this operator is complete in the space $\ell_{w}^{2}(\mathbb{Z})$.

Proof. It can be easily seen from (4.6) that $S_{h_{1} h_{2}}^{-}(\lambda)$ is an inner function in the upper half-plane and, moreover, it is meromorphic in the whole $\lambda$-plane. Then, it can be factorized as

$$
S_{h_{1} h_{2}}^{-}(\lambda)=e^{i \lambda c} B_{h_{1} h_{2}}(\lambda), \quad c=c\left(h_{1}\right) \geq 0,
$$

where $B_{h_{1} h_{2}}(\lambda)$ is a Blaschke product. It can be inferred from (5.1) that

$$
\left|S_{h_{1} h_{2}}^{-}(\lambda)\right| \leq e^{-c\left(h_{1}\right) \operatorname{Im} \lambda}, \quad \operatorname{Im} \lambda \geq 0
$$

Further, if we express $m_{\infty h_{2}}(\lambda)$ in terms of $S_{h_{1} h_{2}}^{-}(\lambda)$ and then use (4.6), we find

$$
m_{\infty h_{2}}(\lambda)=\frac{\bar{h}_{1} S_{h_{1} h_{2}}^{-}(\lambda)-h_{1}}{S_{h_{1} h_{2}}^{-}(\lambda)-1} .
$$

If $c\left(h_{1}\right)>0$ for a given value $h_{1}\left(\operatorname{Im} h_{1}>0\right)$, then $\lim _{t \rightarrow+\infty} S_{h_{1} h_{2}}^{-}(i t)=0$ follows from (5.2). Hence, we obtain $\lim _{t \rightarrow+\infty} m_{\infty h_{2}}(i t)=h_{1}$ in the light of (5.3). Since $m_{\infty} h_{2}(\lambda)$ is independent of $h_{1}, c\left(h_{1}\right)$ can be nonzero at not more than a single point $h_{1}=h_{1}^{0}$ (and, further, $\left.h_{1}^{0}=\lim _{t \rightarrow+\infty} m_{\infty h_{2}}(i t)\right)$. Hence, the theorem is proved.

The proof of the next result is similar to that of Theorem 5.1.

Theorem 5.2. For all values of $h_{2}$ with $\operatorname{Im} h_{2}>0$, except possibly for a single value $h_{2}=h_{2}^{0}$, and for fixed $h_{1}\left(\operatorname{Im} h_{1}=0\right.$ or $\left.h_{1}=\infty\right)$, the characteristic function $S_{h_{1} h_{2}}^{+}(\lambda)$ of the maximal dissipative operator $L_{h_{1} h_{2}}^{+}$is a Blaschke product and the spectrum of $L_{h_{1} h_{2}}^{+}$is purely discrete and belongs to the open upper half-plane. The operator $L_{h_{1} h_{2}}^{+}\left(h_{2} \neq h_{2}^{0}\right)$ has a countable number of isolated eigenvalues with finite algebraic multiplicity and limit points at infinity, and the system of all eigenvectors and associated vectors of this operator is complete in the space $\ell_{w}^{2}(\mathbb{Z})$.

Since a linear operator $\mathbf{S}$ acting in a Hilbert space $\mathbf{H}$ is maximal accretive if and only if $-\mathbf{S}$ is maximal dissipative, all results obtained for maximal dissipative operators can be immediately transferred to maximal accretive operators.

\section{References}

[1] B. Sz-Nagy and C. Foiaş, Analyse Harmonique des Operateurs de L'espace de Hilbert, Masson, Paris, France, 1967.

[2] B. Sz-Nagy and C. Foiaş, Analyse Harmonique des Operateurs de L'espace de Hilbert, North-Holland, Amsterdam, The Netherlands, 1970.

[3] B. S. Pavlov, "Spectral analysis of a dissipative singular Schrödinger operator in terms of a functional model," Itogi Nauki i Tekhniki, vol. 65, pp. 95-163, 1991. 
[4] B. S. Pavlov, "Spectral analysis of a dissipative singular Schrödinger operator in terms of a functional model," in Partial Differential Equations, VIII, M. Shubin, Ed., vol. 65 of Encyclopaedia of Mathematical Sciences, pp. 87-153, Springer, Berlin, Germany, 1996.

[5] P. D. Lax and R. S. Phillips, Scattering Theory, Academic Press, New York, NY, USA, 1967.

[6] B. P. Allakhverdiev and G. Sh. Gusernov, "On the spectral theory of second-order dissipative difference operators," Matematicheskiu Sbornik, vol. 180, no. 1, pp. 101-118, 1989.

[7] B. P. Allakhverdiev and G. Sh. Gusernov, "On the spectral theory of second-order dissipative difference operators," Mathematics of the USSR-Sbornik, vol. 66, no. 1, pp. 107-125, 1990.

[8] B. P. Allahverdiev, "Dissipative second-order difference operators with general boundary conditions," Journal of Difference Equations and Applications, vol. 10, no. 1, pp. 1-16, 2004.

[9] B. P. Allahverdiev, "Extensions, dilations and functional models of infinite Jacobi matrix," Czechoslovak Mathematical Journal, vol. 55, no. 130, pp. 593-609, 2005.

[10] N. I. Akhiezer, The Classical Moment Problem and Some Related Questions in Analysis, Fizmatgiz, Moscow, Russia, 1961.

[11] N. I. Akhiezer, The Classical Moment Problem and Some Related Questions in Analysis, Oliver and Boyd, New York, NY, USA, 1965.

[12] F. V. Atkinson, Discrete and Continuous Boundary Problems, Academic Press, New York, NY, USA, 1964.

[13] M. Benammar and W. D. Evans, "On the Friedrichs extension of semi-bounded difference operators," Mathematical Proceedings of the Cambridge Philosophical Society, vol. 116, no. 1, pp. 167-177, 1994.

[14] Yu. M. Berezanskii, Expansion in Eigenfunctions of Selfadjoint Operators, Naukova, Kiev, Ukraine, 1965.

[15] Yu. M. Berezanskii, Expansion in Eigenfunctions of Selfadjoint Operators, American Mathematical Society, Providence, RI, USA, 1968.

[16] J. Chen and Y. Shi, "The limit circle and limit point criteria for second-order linear difference equations," Computers \& Mathematics with Applications, vol. 47, no. 6-7, pp. 967-976, 2004.

[17] S. T. Welstead, "Boundary conditions at infinity for difference equations of limit-circle type," Journal of Mathematical Analysis and Applications, vol. 89, pp. 442-461, 1982.

[18] R. P. Agarwal, Difference Equations and Inequalities, vol. 228 of Revised and Expanded, Marcel Dekker, New York, NY, USA, 2nd edition, 2000.

[19] S. N. Elaydi, An Introduction to Difference Equations, Springer, New York, NY, USA, 1996. 


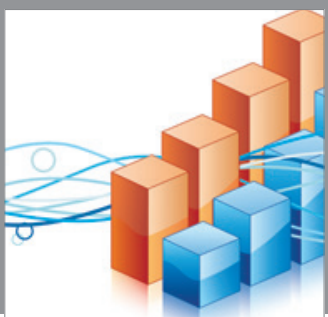

Advances in

Operations Research

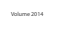

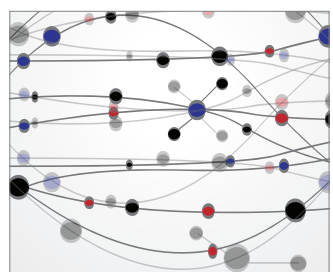

\section{The Scientific} World Journal
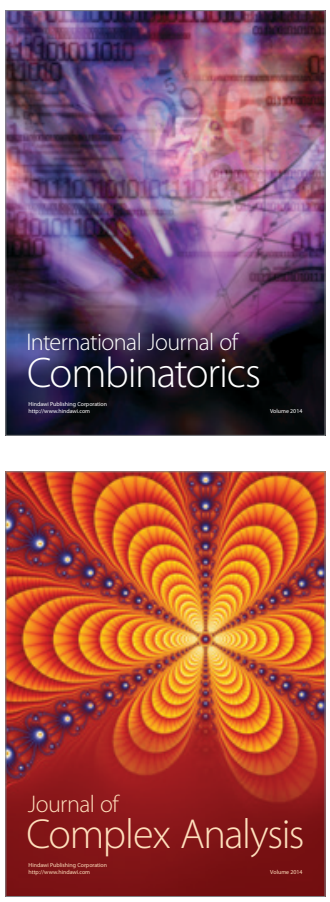

International Journal of

Mathematics and

Mathematical

Sciences
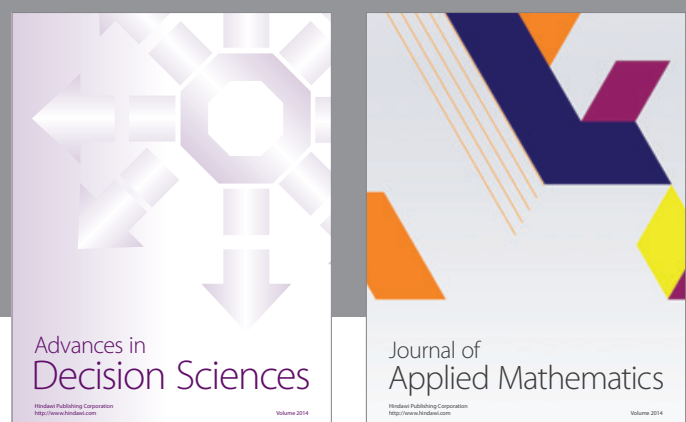

Journal of

Applied Mathematics
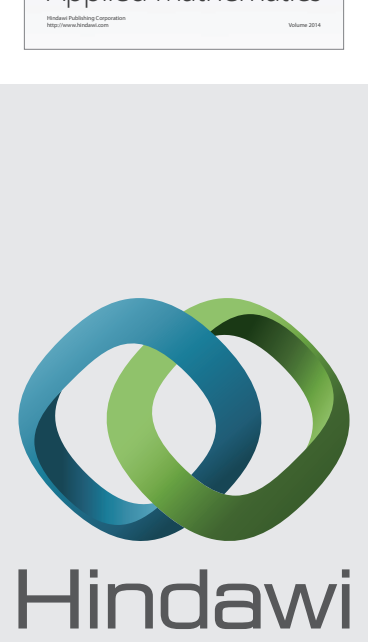

Submit your manuscripts at http://www.hindawi.com
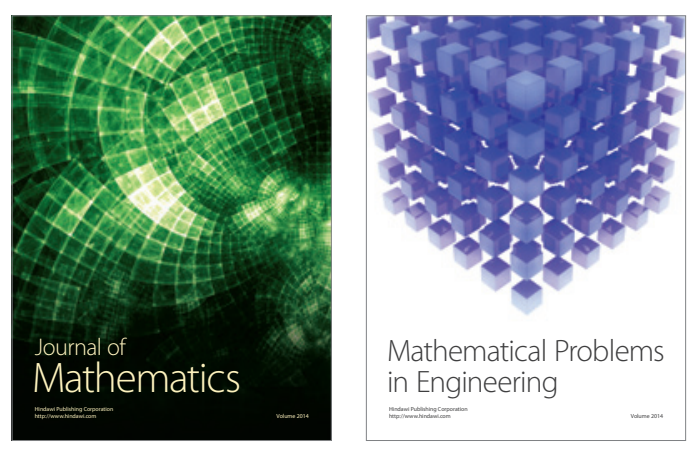

Mathematical Problems in Engineering
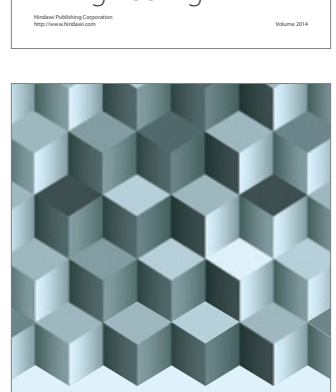

Journal of

Function Spaces
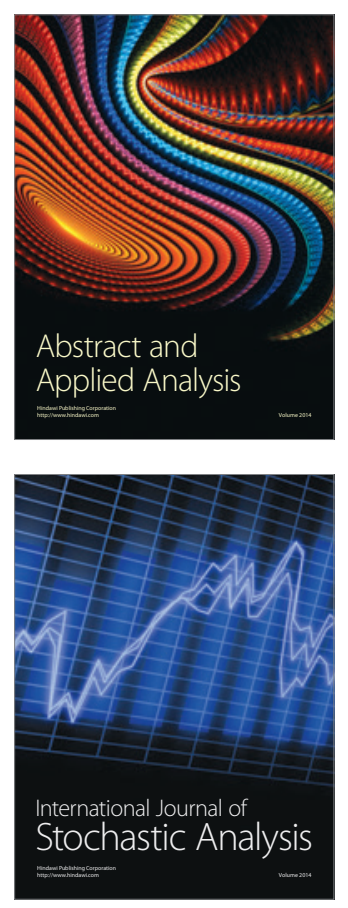

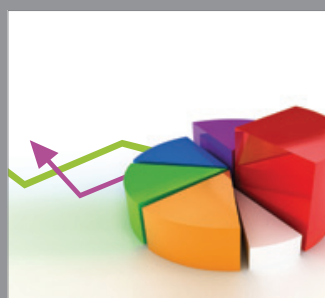

ournal of

Probability and Statistics

Promensencen
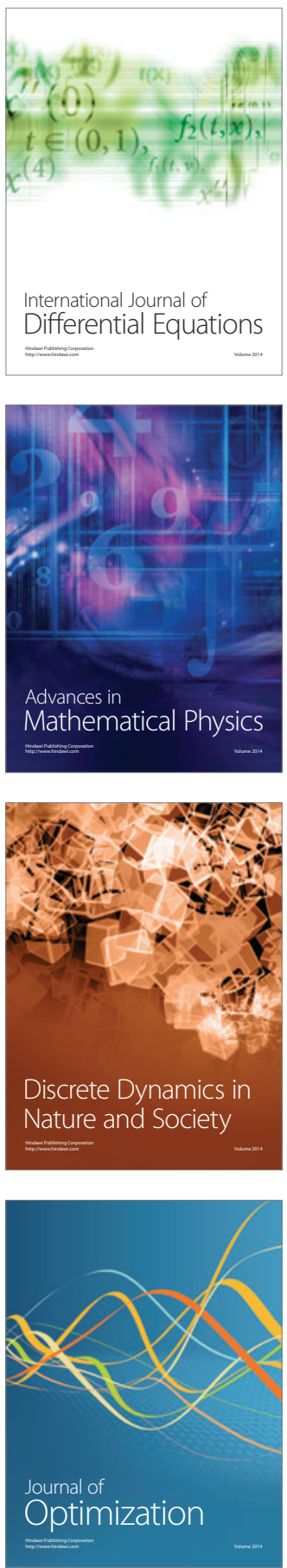